\title{
Functional Specificity of Claustrum Connections in the Rat: Interhemispheric Communication between Specific Parts of Motor Cortex
}

\author{
Jared B. Smith and Kevin D. Alloway \\ Department of Neural and Behavioral Sciences, Pennsylvania State University College of Medicine, Hershey, Pennsylvania 17033-2255
}

\begin{abstract}
Recent evidence indicates that the rat claustrum interconnects the motor cortical areas in both hemispheres. To elucidate the functional specificity of the interhemispheric connections between the claustrum and primary motor (MI) cortex, anterograde tracer injections in specific parts of MI were paired with retrograde tracer injections in homotopic sites of the opposite hemisphere. In addition to injecting the MI forepaw (Fp) region in both hemispheres, we injected the region associated with whisker retractions $(\mathrm{Re})$ and the more caudal rhythmic whisking (RW) region. While the MI-Fp region has few connections with the claustrum of either hemisphere, both whisker regions project to the contralateral claustrum, with those from the MI-RW region being denser and more extensive than those originating from the MI-Re region. Retrograde tracer injections in the MI-RW region produced more labeled neurons in the ipsilateral claustrum than retrograde tracer injections in the MI-Re. Consistent with these patterns, the overlap of labeled terminals and soma in the claustrum was greatest when both tracers were injected into the MI-RW region. When retrograde tracers were injected into the claustrum, the highest density of labeled neurons in MI appeared in the contralateral RW region. Tracer injections in the claustrum also revealed hundreds of labeled neurons throughout its rostrocaudal extent, thereby establishing the presence of long-range intraclaustral connections. These results indicate that the intrinsic and extrinsic connections of the rat claustrum are structured for rapid, interhemispheric transmission of information needed for bilateral coordination of the MI regions that regulate whisker movements.
\end{abstract}

\section{Introduction}

The claustrum is an elongated neuronal structure that extends rostrocaudally through the telencephalon of all mammals including rats (Kowianski et al., 1999). The claustrum receives inputs from multiple cortical areas (Pearson et al., 1982; Carey and Neal, 1985; Clarey and Irvine, 1986), but multimodal integration is minimal because separate parts of the claustrum processes different modalities (Olson and Graybiel, 1980; Remedios et al., 2010). Despite this topographic organization, many neurons in the claustrum project to widely separated cortical targets (Minciacchi et al., 1985; Li et al., 1986). These and other findings indicate that the claustrum may coordinate widespread cortical regions for specific behavioral and cognitive functions (Edelstein and Denaro, 2004; Crick and Koch, 2005).

Consistent with its role in orchestrating neural processes in separate cortical areas, the claustrum has connections with the motor cortices in both hemispheres (Minciacchi et al., 1985; Li et al., 1986; Sloniewski et al., 1986). In cats, electrical stimulation of the claustrum excites the frontal eye fields in both hemispheres, and this suggests that the claustrum could synchronize cortical

Received Aug. 24, 2010; revised 0ct. 5, 2010; accepted 0ct. 18, 2010.

This work was supported by National Institutes of Health Grant NS37532. We thank Jon Harrold and Kyle Beauchemin for assistance with the histology. Jon Harrold also helped with marking labeled varicosities in the confocal images.

Correspondence should be addressed to Dr. Kevin D. Alloway, Neural and Behavioral Sciences, H109, Hershey Medical Center, 500 University Drive, Hershey, PA 17033-2255. E-mail: kda1@psu.edu.

DOI:10.1523/JNEUROSCI.4438-10.2010

Copyright $\odot 2010$ the authors $\quad 0270-6474 / 10 / 3016832-13 \$ 15.00 / 0$ regions that subserve bilaterally coordinated behaviors such as eye movements (Cortimiglia et al., 1991). Like eye movements, rats actively move their whiskers on both sides of the face, and these simultaneous movements are bilaterally coordinated during different behavioral situations (Towal and Hartmann, 2006; Mitchinson et al., 2007). In view of the bilateral nature of eye and whisker movements, similar claustral circuits might mediate the interhemispheric coordination that regulates these behaviors.

Our laboratory has shown that the rat claustrum receives bilateral projections from the whisker region in primary motor (MI) cortex, but the contralateral projections are more prominent (Alloway et al., 2009). Furthermore, the rat claustrum projects to the ipsilateral MI whisker region but does not project to the contralateral hemisphere (Colechio and Alloway, 2009). In comparison, the MI forepaw region has few afferent or efferent connections with the claustrum in either hemisphere. These results suggest that one function of the claustrum and its interhemispheric connections is to coordinate the motor areas that regulate specific types of bilateral movements.

The present study assessed the plausibility of this view by injecting an anterograde tracer into motor cortex of one hemisphere and a retrograde tracer into the corresponding motor region of the other hemisphere. This bilateral two-tracer approach tested the hypothesis that corticoclaustral and claustrocortical neurons form interhemispheric circuits between specific motor regions. In addition to injecting the motor forepaw (MI$\mathrm{Fp}$ ) regions in one group of rats, two other groups received tracer injections in motor regions that evoke specific types of whisker 
movements. One whisker region, located in medial agranular cortex, evokes whisker retractions (MI-Re) when microstimulated (Brecht et al., 2004). The second, more caudal whisker region evokes protractions or rhythmic (5-15 Hz) whisking (MI-RW) movements when microstimulated (Sanderson et al., 1984; Haiss and Schwarz, 2005). The results revealed a corticoclaustro-cortical interhemispheric circuit that interconnects the MI whisker regions of both hemispheres, especially the RW regions.

\section{Materials and Methods}

All procedures on adult male Sprague Dawley rats (Charles River) conformed to National Institutes of Health guidelines and were approved by our Institutional Animal Care and Use Committee.

Animal surgery. Rats were initially anesthetized by an intramuscular injection of ketamine $\mathrm{HCl}(20 \mathrm{mg} / \mathrm{kg})$ and xylazine $(6 \mathrm{mg} / \mathrm{kg})$, followed by intramuscular injections of atropine methyl nitrate $(0.5 \mathrm{mg} / \mathrm{kg})$, dexamethasone sodium phosphate $(5 \mathrm{mg} / \mathrm{kg}$ ), and chloramphenicol $(50 \mathrm{mg} /$ $\mathrm{kg}$ ). Each rat was intubated, placed in a stereotaxic frame, and ventilated with oxygen. A homeothermic heating blanket maintained body temperature at $37^{\circ} \mathrm{C}$, ophthalmic ointment was used to prevent corneal drying, and vital signs such as heart rate, specific oxygen, and end-tidal carbon dioxide (Surgivet) were monitored continuously. A midline incision was made over the cranium, and lidocaine (2\%) was injected into the wound margins. Craniotomies were made bilaterally over the motor cortex of each hemisphere at coordinates consistent with previous studies (Hall and Lindholm, 1974; Hoffer et al., 2003; Brecht et al., 2004; Haiss and Schwarz, 2005).

Tracer injections in MI. Intracortical microstimulation (ICMS) was administered to MI cortex as short $(80 \mathrm{~ms})$ and long $(1400 \mathrm{~ms})$ pulse trains by a glass pipette filled with hypertonic ( $3 \mathrm{M})$ saline (0.4-1.3 $\mathrm{M} \Omega$ ). Short stimulus trains were administered at $250 \mathrm{~Hz}(0.7 \mathrm{~ms}$ pulses separated by intervals of $3.3 \mathrm{~ms}$ ), whereas long stimulus trains were tested at both 250 and $100 \mathrm{~Hz}(0.7 \mathrm{~ms}$ pulses separated by intervals of $9.3 \mathrm{~ms})$. The MI cortex was briefly mapped to identify sites that evoked movements of the forepaw or whiskers. Mapping of MI commenced when the rat spontaneously began making just-noticeable whisker movements because this indicated an anesthetic plane that allowed ICMS to evoke muscle twitches.

The microstimulation electrode entered cortex orthogonal to the pial surface, and ICMS was administered at depths of $1.0-1.7$, which is where corticospinal and corticobulbar projections originate. Multiple depths were stimulated along the electrode penetration, especially in the medial bank of MI where the functional topography can change suddenly [Brecht et al. (2004), their Fig. 3]. Short ICMS trains at low currents $(<50 \mu \mathrm{A})$ evoked brief dorsoflexion of the forepaw in the MI-Fp region, whisker retractions in the MI-Re region, and whisker protractions in the MI-RW region. In contrast, long ICMS trains in the MI-Fp region produced sustained contraction of the forelimb muscles, sustained retraction of the whiskers in the MI-Re region, and low-amplitude $(\sim 2-3 \mathrm{~mm})$ rhythmic $(5-15 \mathrm{~Hz})$ whisking movements in the MI-RW region. These observations are consistent with other reports (Sanderson et al., 1984; Haiss and Schwarz, 2005). Mapping started in the MI-Re region and continued caudally until the MI-RW region was located and then continued laterally and rostrally to locate the MI-Fp region. Stimulation sites were separated by at least $500 \mu \mathrm{m}$, and four to seven sites were tested in each hemisphere so that tracer injections were $>500 \mu \mathrm{m}$ away from ICMS sites that had different functional representations.

After locating a suitable injection site, the stimulating electrode was removed and an injection pipette was inserted at that site. Tracer injections were made bilaterally in the Fp, Re, or RW regions of MI at depths of 1.1-1.7 mm below the pial surface. In one hemisphere, a $2 \%$ solution (in physiologic saline) of the retrograde tracer Fluoro-Gold (FG; $\mathrm{H}-22845$; Fluoro-Chrome) was iontophoretically administered through a glass pipette (30-60 $\mu \mathrm{m}$ outer tip) at $2 \mu \mathrm{A}$ for $15 \mathrm{~min}$ using a $7 \mathrm{~s}$ on/off duty cycle. The other hemisphere was pressure injected with $100 \mathrm{nl}$ of a $15 \%$ solution (in $0.01 \mathrm{~m} \mathrm{PBS}$ ) of the anterograde tracer Fluoro-Ruby (FR; D-1817; Invitrogen) by means of a Hamilton syringe with a glass pipette (60-80 $\mu \mathrm{m}$ tip) cemented on the end. As shown in Table 1, tracer injec-
Table 1. Summary of bilateral tracer injections

\begin{tabular}{cllllll}
\hline & & \multicolumn{2}{l}{ Left hemisphere } & & \multicolumn{2}{l}{ Right hemisphere } \\
Case & Region & Tracer & Coordinates $(\mathrm{R}, \mathrm{L})$ & & Tracer & Coordinates $(\mathrm{R}, \mathrm{L})$ \\
\hline CL01 & MI-Re & FR & $2.3,1.2$ & & FG & $2.3,1.5$ \\
CL02 & MI-Re & FR & $2.7,1.3$ & & FG & $2.5,1.4$ \\
CL03 & MI-Fp & FR & $2.9,2.8$ & & FG & $2.8,2.7$ \\
CL04 & MI-Fp & FR & $2.5,2.3$ & & FG & $2.7,2.5$ \\
CL05 & MI-Re & FR & $2.3,1.4$ & & FG & $2.3,1.3$ \\
CL06 & MI-Fp & FR & $2.5,2.7$ & & FG & $2.6,2.8$ \\
CL21 & MI-RW & FR & $1.6,1.0$ & & FG & $1.6,0.9$ \\
CL22 & MI-RW & FR & $1.7,1.0$ & & FG & $1.7,1.0$ \\
CL23 & MI-RW & FR & $1.6,1.2$ & FG & $1.4,1.1$ \\
\hline
\end{tabular}

Coordinates are rostral $(\mathrm{R})$ and lateral $(\mathrm{L})$ to bregma in millimeters.

tions for each functional region in MI were placed in a limited range of stereotaxic coordinates (in mm): MI-Fp region, 2.5-2.9 rostral and 2.32.8 lateral to bregma; MI-Re region, 2.3-2.7 rostral and 1.2-1.5 lateral to bregma; MI-RW region, 1.4-1.7 rostral and 0.9-1.2 lateral to bregma.

Retrograde tracer injections in claustrum. In a separate group of rats, the retrograde tracer (FG) was iontophoretically injected into the claustrum or surrounding regions. After an incision was made along the face between the eye and the ear, the tissue was retracted to allow access to the skull just caudal to the orbital socket $(1.4 \mathrm{~mm}$ rostral to bregma and 6.9 $\mathrm{mm}$ ventral to the surface of the skull at bregma). After a craniotomy, a glass pipette oriented orthogonal to the temporal bone (i.e., parallel to the stereotaxic ear bars) was inserted into the brain while a retention current $(-5 \mu \mathrm{A})$ was applied to prevent unwanted labeling of the insular cortex. The retrograde tracer was then injected at a depth of $1.75 \mathrm{~mm}$ from the pial surface with $+5 \mu \mathrm{A}$ pulses for 20 min using a $7 \mathrm{~s}$ on/off duty cycle. Compared with MI injections, larger currents were needed for FG injections into the claustrum because the pipette was oriented horizontally and gravitational force could not facilitate tracer expulsion into the claustrum. The retention current was reapplied while withdrawing the pipette.

Histology. After tracer injections, the wound margins were sutured, and the rat received supplemental doses of atropine, dexamethasone, and chloramphenicol before it was returned to its home cage for $7 \mathrm{~d}$. After this, each rat was deeply anesthetized with sodium pentobarbital (100 $\mathrm{mg} / \mathrm{kg}$, i.p.) and perfused transcardially with saline, $4 \%$ paraformaldehyde, and $4 \%$ paraformaldehyde with $10 \%$ sucrose. The brain was removed and refrigerated in $4 \%$ paraformaldehyde with $30 \%$ sucrose for $2-3 \mathrm{~d}$. The olfactory bulbs and cerebellum were removed, and a small slit was made in the left hemisphere, ventral to the rhinal fissure, to distinguish the two sides of the brain. The brain was sectioned coronally (60 $\mu \mathrm{m}$ ), and sections were saved in $0.1 \mathrm{M}$ PBS. Serially ordered sections were used to make three series, one for fluorescent labeling, one for thionin staining, and one for processing cytochrome oxidase. Sections from the fluorescent series were mounted on gelatin-coated glass slides and dried overnight in an incubator. The next day, these sections were dehydrated in $100 \%$ ethanol, defatted in xylene, and coverslipped with Cytoseal. The remaining two series, which were used to reveal cytoarchitectonic borders, were stained with thionin or processed for the presence of cytochrome oxidase (Land and Simons, 1985).

Anatomical analysis. Alternate sections in the fluorescent series, which were obtained at intervals of $360 \mu \mathrm{m}$ during frozen sectioning, were used to reconstruct the locations of labeled terminals and neurons. The remaining intervening sections in the fluorescent series were used for photomicrography of the labeling patterns. The locations of labeled varicosities and neurons were reconstructed with a BH-2 microscope (Olympus) equipped with a reconstruction system (AccuStage) in which optical transducers were mounted on the $x--y$ axes of the microscope stage to construct digital records of FR-labeled terminal varicosities and FG-labeled neuronal soma, as well as other anatomical features (MDPlot software, version 5.1; AccuStage.com). The microscope contained a tetrarhodamine isothiocyanate filter (41002; Chroma Technologies) for visualizing the axonal varicosities of the FR-labeled terminal arbors and a UV filter (51004v2; Chroma Technologies) for visualizing FG-labeled neuronal soma. Plotting of fluorescent labeling was done with a $10 \times$ 

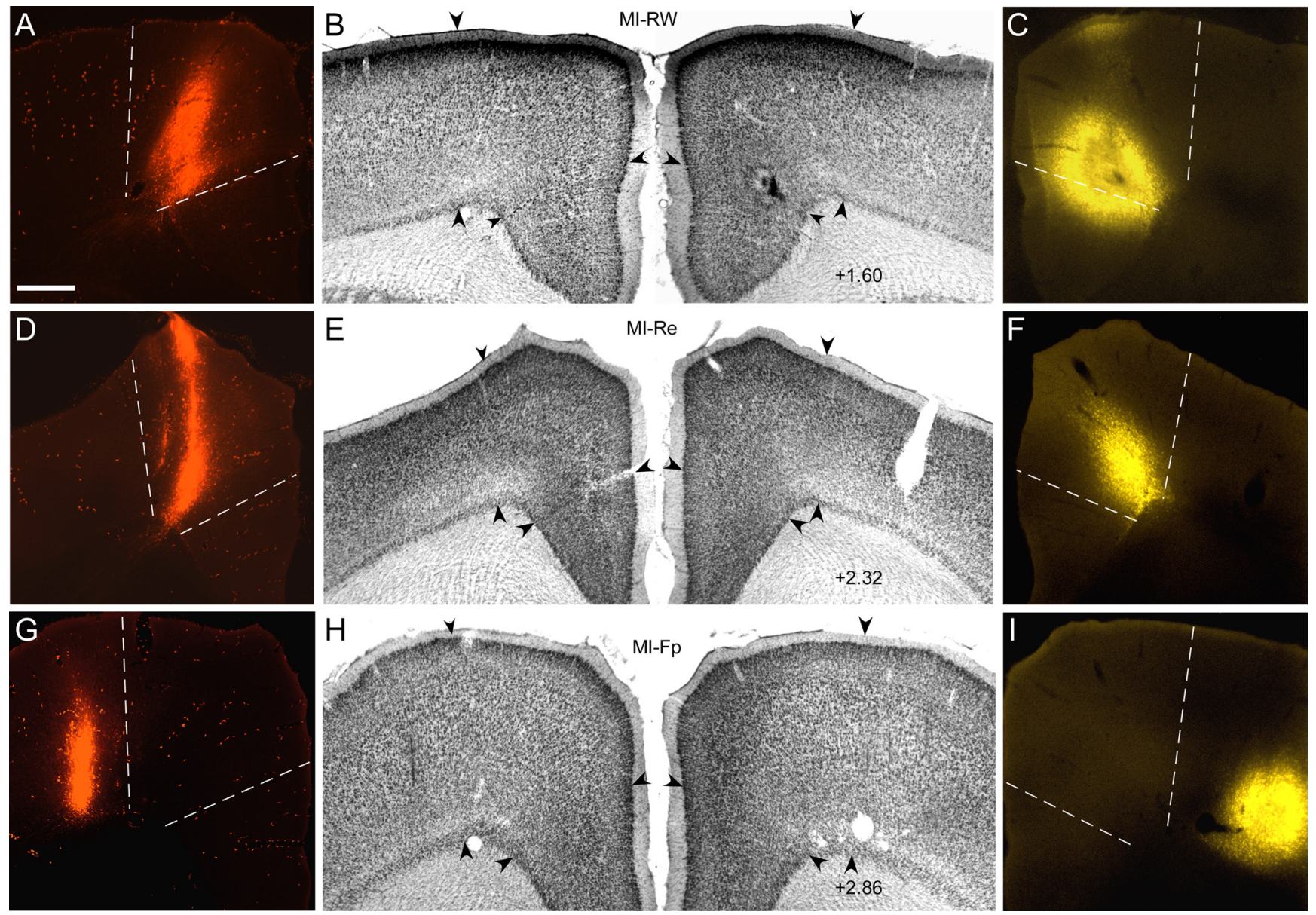

Figure 1. Representative examples of bilateral injections of FR and FG into the RW, Re, and Fp regions of MI cortex. $A$, FR injection in the RW region of the left hemisphere of case CL21. $\boldsymbol{B}$, Thionin-stained section depicting MI cortical cytoarchitecture at the level of the tracer injections. C, FG injection in the RW region of the right hemisphere. D-I, FR and FG injections in the Re (CL05) and $\mathrm{Fp}$ (CLO3) regions depicted as in $\boldsymbol{A}-\boldsymbol{C}$. Dashed lines and arrowheads indicate the cytoarchitectonic borders of the medial Agm cortex. Numbers (bottom right) in $\boldsymbol{B}, \boldsymbol{E}$, and $\boldsymbol{F}$ indicate distance from bregma. Scale bar, $0.5 \mathrm{~mm}$ for all panels.

eyepiece in combination with a $10 \times$ or $20 \times$ objective. For each reconstruction, the anterogradely labeled (FR) terminals were plotted first. Beaded varicosities along the labeled axons were plotted because axonal enlargements are known to contain synaptic vesicles that are colocalized with markers for synaptophysin, and thus represent en passant synapses (Voigt et al., 1993; Kincaid and Wilson, 1996; Meng et al., 2004). Subsequently, the filters were switched so that the retrogradely labeled (FG) soma could be plotted in the same claustral area.

Based on the identification of a protein, Gng2, that is expressed in the claustrum and distinguishes it from adjacent structures (Mathur et al., 2009), analysis of claustral labeling did not extend beyond the most rostral coronal section that contained the striatum because more rostral regions do not express Gng2. Neuronal labeling (cell bodies or terminal varicosities) was plotted in sections located caudal to the rostral edge of the striatum if it appeared adjacent to the external capsule at the level of the rhinal sulcus. Tracer injection sites, neuronal labeling, and cytoarchitecture were documented by images acquired with a Retiga EX CCD digital camera (Q-imaging) mounted on the Olympus light microscope.

Plotted reconstructions were analyzed for the number of labeled varicosities and labeled soma, the areal extent of labeling, and the spatial overlap of labeled terminals and cell bodies. For these purposes, the reconstructions were converted into color-coded density diagrams in which the number of plotted processes (neurons or varicosities) within a spatial unit (e.g., 25, 50, or $100 \mu \mathrm{m}^{2}$ ) could be represented by a specific color. These procedures were performed by specific modules in the MDPlot software (version 5.1; AccuStage)

The density of the terminal varicosities varied substantially across sections and from one region to another within the claustrum. In places where terminal labeling was sparse to moderate, the AccuStage system enabled accurate reconstructions of varicosity density. In more densely labeled regions, however, each labeled varicosity could not be individually visualized and plotted using conventional light microscopy. Therefore, an accurate measurement of the density of anterogradely labeled varicosities in the most densely labeled regions required confocal microscopy. For this purpose, a confocal microscope (TCS SP2 AOBS; Leica Microsystems) equipped with a $63 \times$ oil-immersion objective was used with an optical zoom $(120 \times)$ to acquire high-resolution images of both anterogradely labeled (FR) terminals (543 nm excitation, $565-630 \mathrm{~nm}$ detection) and retrogradely labeled (FG) neurons (405 nm excitation, $520-600 \mathrm{~nm}$ detection) in the claustrum.

To determine the maximum density of anterogradely labeled varicosities in the claustrum, a square region $\left(125 \mu \mathrm{m}^{2}\right)$ or counting box was obtained from the center of the ventral claustrum as defined by Paxinos and Watson (2005). Multiple images were taken through the depth of the section, such that the final image contained a sum of luminance values for each pixel extending $15 \mu \mathrm{m}$ through the $z$-plane (maximum projection). These images were imported into a software drawing program (Canvas X; Deneba Systems), and two individuals (K.D.A. and J.H.) blind to the experimental details for each image were responsible for counting the number of labeled varicosities. For this procedure, a green dot was placed on each anterogradely labeled varicosity, and, when finished, the drawing program counted the number of green dots placed on the image. This produced an accurate count of the labeled varicosities, which was then used to calculate their density in this focal region. Consistent with previous criteria for calculating the density of en passant axonal varicosities (Reep et al., 2008; Alloway et al., 2010), only axonal 

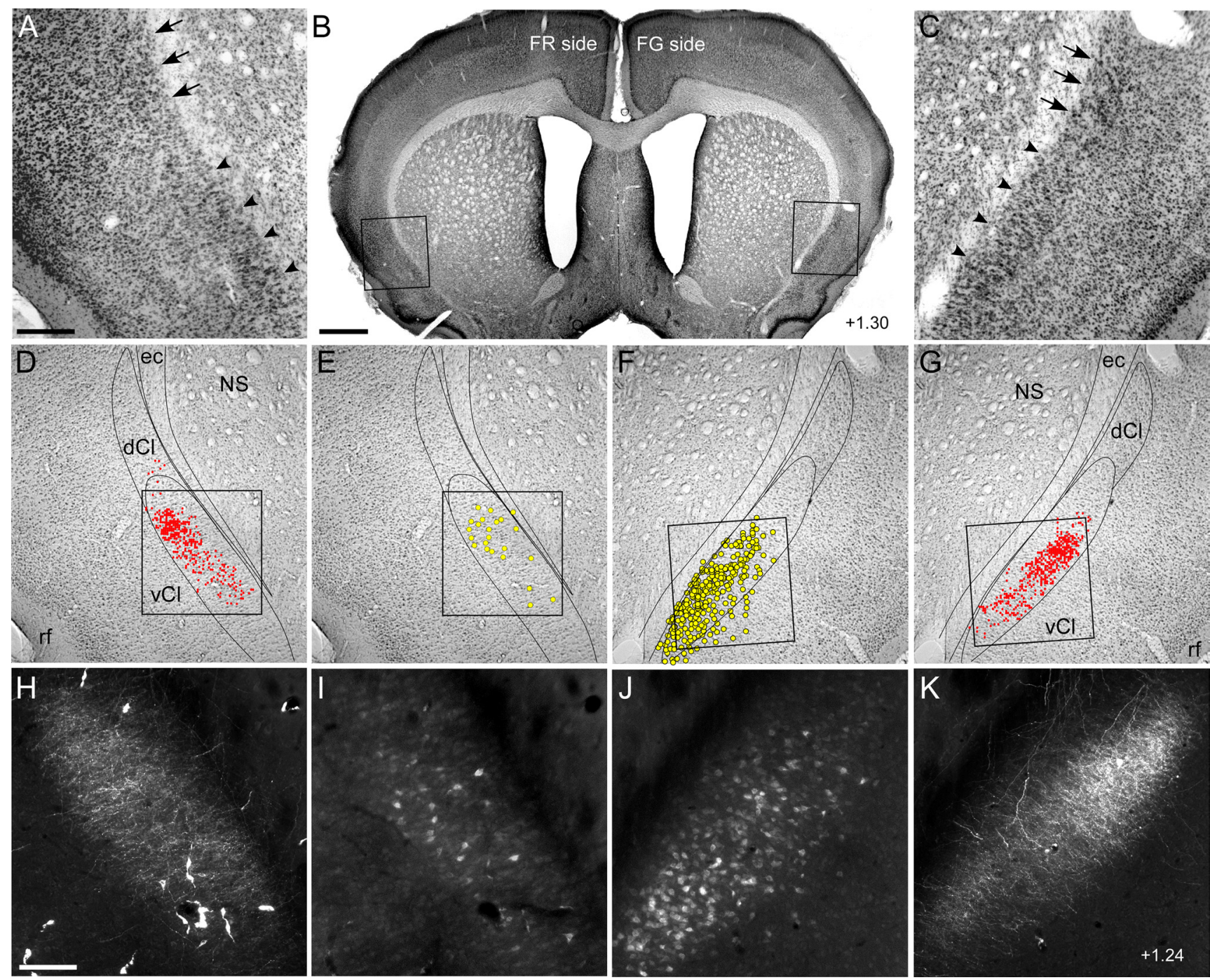

Figure 2. Bilateral labeling patterns in the claustrum produced by FR and FG injections in the MI-RW regions of CL21 (see Fig. $1 A, C$. $A, C$, Magnified views of the left and right claustrum; dorsal and ventral subnuclei are indicated by arrows and arrowheads, respectively. $\boldsymbol{B}$, Coronal section indicating the claustral areas (rectangles) depicted in $\boldsymbol{A}$ and $\boldsymbol{C}$. As indicated, FR was injected on the left, and FG was injected on the right. The number indicates the distance from bregma. $\boldsymbol{D}-\mathbf{G}$, Claustral boundaries and plotted locations of FR-labeled varicosities ( $\boldsymbol{D}, \mathbf{G}$, small red dots) and FG-labeled neurons $(\boldsymbol{E}, \boldsymbol{F}$, large yellow dots). Boxes indicate regions depicted in the last row. $\boldsymbol{H}-\boldsymbol{K}$, Photomicrographs of FR-labeled terminals $(\boldsymbol{H}, \boldsymbol{K})$ and $F G$-labeled neurons $(\boldsymbol{I}, \boldsymbol{J})$ in the claustrum. The number in $\boldsymbol{K}$ indicates distance from bregma. Scale bars: $\boldsymbol{A}, \boldsymbol{C}-\mathbf{G}, 250 \mu \mathrm{m} ; \boldsymbol{B}, 1 \mathrm{~mm} ; \boldsymbol{H}-\boldsymbol{K}, 100 \mu \mathrm{m}$. ec, External capsule; dCl, dorsal claustrum; NS, neostriatum; rf, rhinal fissure; $\mathrm{vCl}$, ventral claustrum.

enlargements twice the diameter of the emerging axon on each side were counted.

Differences in terminal and neuronal labeling were analyzed with several statistical procedures including ANOVA, as well as paired and independent $t$ tests. The statistical computations and determination of significance levels were performed by modules in Origin software (version 8.0; OriginLab).

\section{Results}

The bilateral connections between MI and the claustrum were analyzed in all nine rats that received bilateral tracer injections. As shown in Table 1, injections in each rat were bilaterally placed in homotopic sites located in the MI-Fp $(n=3)$, MI-Re $(n=3)$, or MI-RW $(n=3)$ regions as defined by movements evoked by short ( $80 \mathrm{~ms}$ ) and long (1400 ms) trains of ICMS. Figure 1 illustrates examples of bilateral tracer injections in the RW, Re, and Fp regions of MI. Photomicrographs of the tracer injections were compared with photomicrographs of adjacent thionin-stained sections to locate the injection site with respect to the cytotectonic boundaries of MI cortex. Consistent with previous re- ports (Brecht et al., 2004; Alloway et al., 2008, 2009), tracer injections in MI-Re were always in medial agranular (Agm) cortex, whereas injections in MI-Fp were always in lateral agranular (Agl) cortex.

Tracer injections in sites that evoked rhythmic whisking movements were concentrated in the part of Agm cortex that is closest to the cingulate $(\mathrm{Cg})$ cortex. Iontophoretic administration of the retrograde tracer (FG) produced sphere-shaped deposits in which the ventral portion of the tracer injection crossed the boundary between Agm and Cg cortices. Microstimulation of this part of Cg cortex evokes eye movements (Brecht et al., 2004), but we did not observe eye twitches during our MI mapping procedures. Compared with maps reported by Brecht et al. (2004), in which eye twitches were evoked by ICMS at depths 2 $\mathrm{mm}$ and deeper from the pial surface, ICMS in our study was administered at depths of $\leq 1.7 \mathrm{~mm}$, and this explains why eye twitches were not observed in our study. Nonetheless, the ventral portion of our retrograde tracer injections in the RW region extended into the dorsal part of the underlying Cg cortex. 

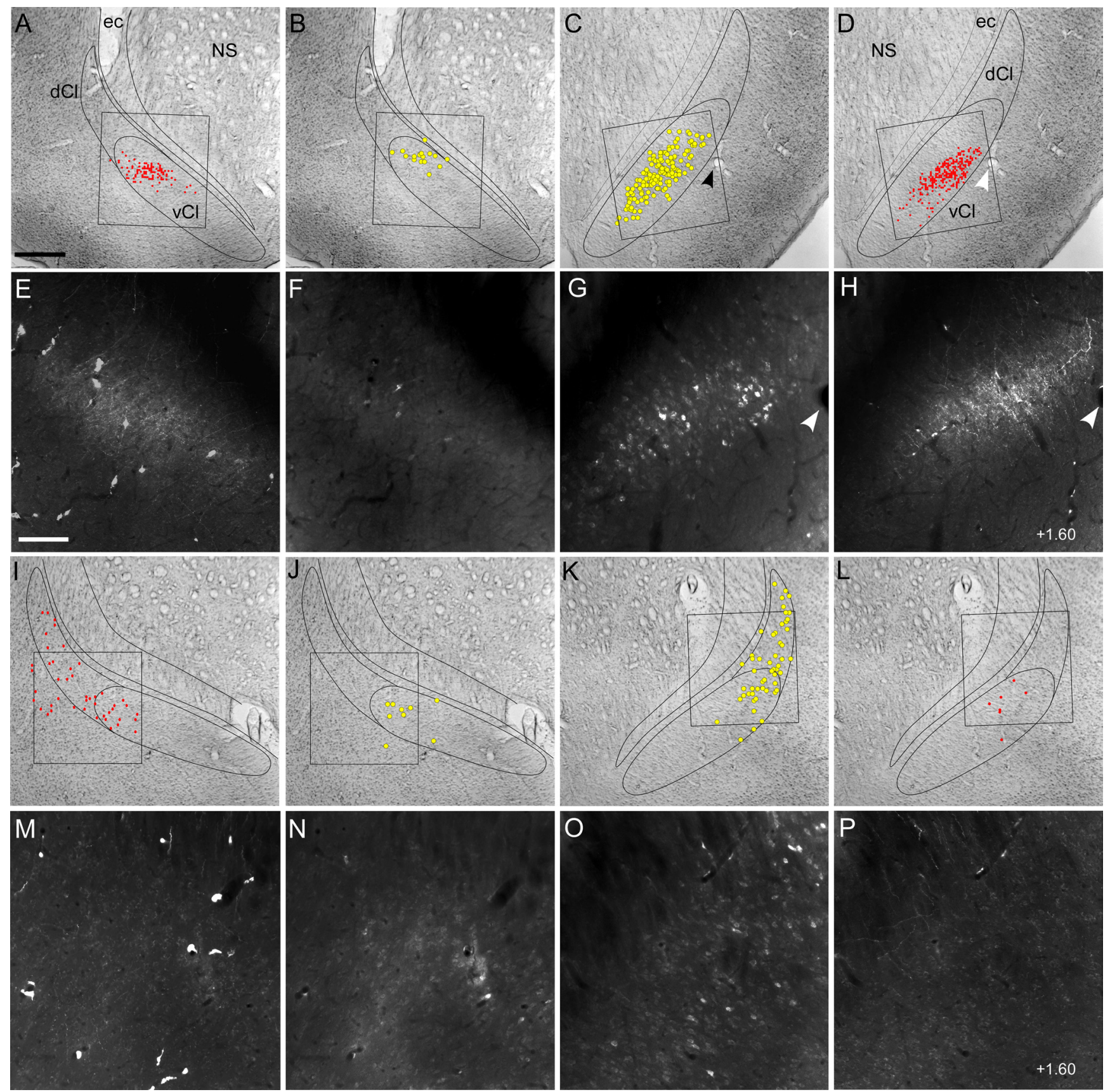

Figure 3. Bilateral labeling patterns in the claustrum produced by FR and FG injections in the Re (CL05) and Fp (CL03) regions of MI (see Fig. 1). Claustral labeling is displayed as in Figure 2D-K. $\boldsymbol{A}-\boldsymbol{H}$, Plotted reconstructions and photomicrographs of FR-labeled varicosities and FG-labeled neurons in the claustrum after tracer injections in MI-Re. I-P, Reconstructions and photomicrographs of a small amount of claustral labeling after tracer injections in MI-Fp. Large FR-labeled cells in $\boldsymbol{E}$ and $\boldsymbol{M}$ are pericytes associated with blood vessels.

In comparison, pressure injections of FR caused this anterograde tracer to flow upward along the injection pipette, thereby forming an elongated tracer deposit that extended toward the superficial layers of MI. Although the ventral edge of the FR injections approached the medial border of Agm, very little, if any, of the tracer diffused into Cg cortex (Fig. 1A).

\section{Location of claustral labeling}

The nomenclature for the claustrum has changed over the past two decades, and most of the labeling that we observed in the claustrum was concentrated in the ventral subnucleus as outlined in a recent atlas (Paxinos and Watson, 2005). As shown in Figure 2 , the ventral claustrum is characterized by an oval-shaped region of small to medium neurons that is capped by a small region of densely packed neurons that form the dorsal claustrum. Located dorsal to the deep endopiriform nucleus, the ventral claustrum adjoins the part of the external capsule that becomes noticeably narrower as it proceeds ventrally near the rhinal fissure.

Recent evidence indicates that the neuropil in the rat claustrum is associated with a membrane-linked receptor protein known as Gng2 (Mathur et al., 2009). This protein is not expressed in sections located beyond the rostral edge of the striatum, and, consistent with this pattern of protein expression, the amount of labeling declined steeply in sections located immediately rostral to the striatum. Therefore, our analysis of anterograde and retrograde labeling in the claustrum commenced at the 

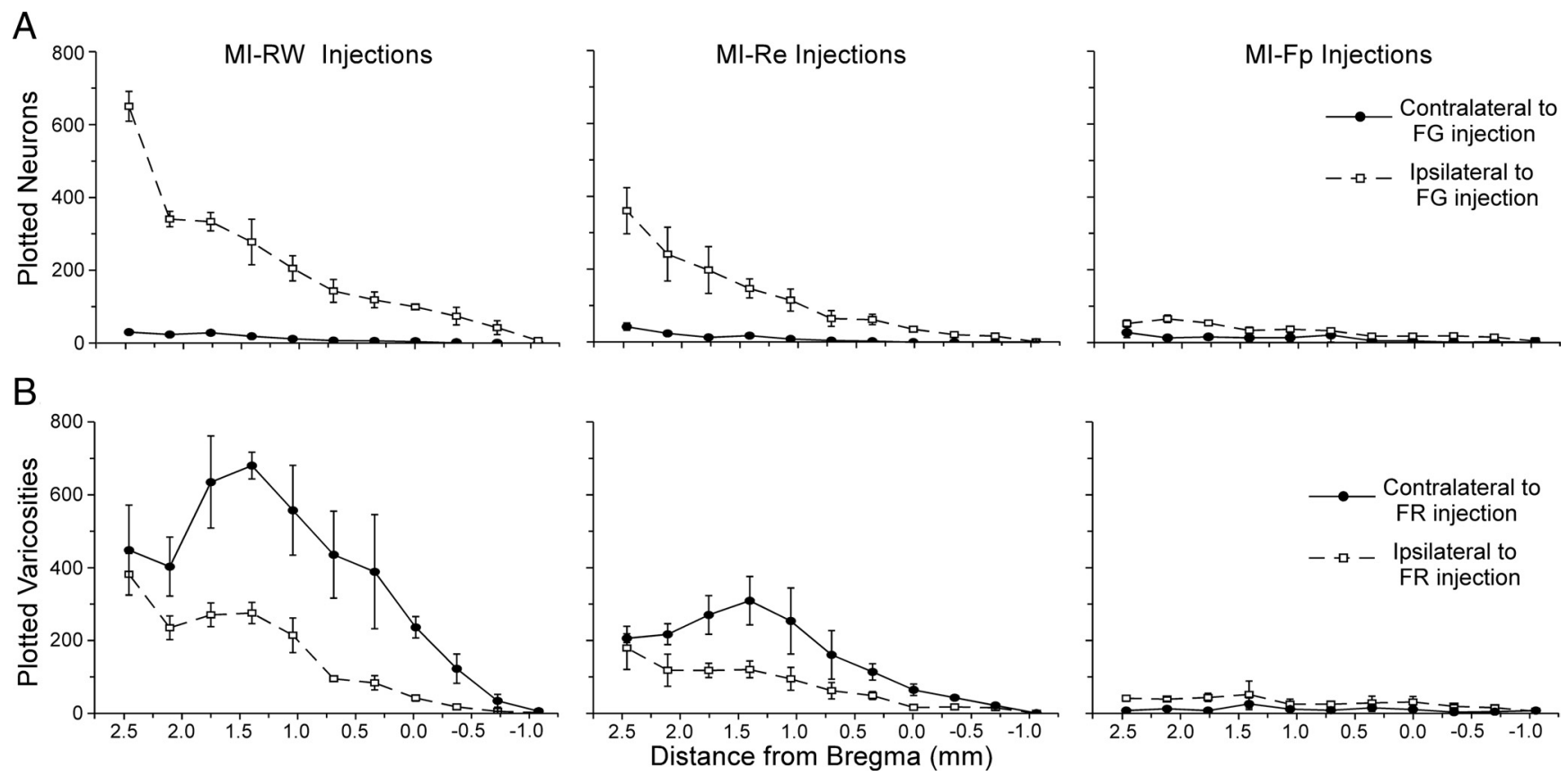

Figure 4. Rostrocaudal profile of the bilateral distribution of claustral labeling produced by tracer injections in the RW, Re, and Fp regions of MI. $A$, Distribution of FG-labeled neurons plotted contralateral and ipsilateral to the FG injection site. $\boldsymbol{B}$, Distribution of FR-labeled varicosities plotted contralateral and ipsilateral to the FR injection site. Symbols represent the mean number of plotted processes at each rostrocaudal position; error bars represent SEM.

first coronal section that contained the rostral striatum $(\sim 2.5$ $\mathrm{mm}$ rostral to bregma) and continued caudally until claustral labeling was no longer present.

In the nine rats that received bilateral tracer injections in MI cortex, labeling in the claustrum consistently appeared within a rostrocaudal range that extended from $2.5 \mathrm{~mm}$ rostral to $1.4 \mathrm{~mm}$ caudal to bregma. Because reconstructions were performed on coronal sections obtained at intervals of $360 \mu \mathrm{m}$ (see Materials and Methods), exactly 11 sections within this rostrocaudal range were plotted in each rat.

\section{Functional specificity of claustral labeling}

Our bilateral tracer injections in the MI-RW regions produced distinct asymmetric patterns of claustral labeling across the two hemispheres. As indicated in Figure 2, an injection of the retrograde tracer (FG) into the RW region of the right hemisphere labeled many neuronal soma in the right claustrum but labeled very few neurons in the left claustrum. In the same rat, an injection of the anterograde tracer (FR) into the RW region of the left hemisphere produced dense terminal labeling in the right claustrum but noticeably less terminal labeling in the left claustrum. Hence, the claustrum located ipsilateral to the retrograde tracer (FG) injection contained a sizeable region in which high densities of FR-labeled terminals and FG-labeled soma were intermingled in overlapping areas. In contrast, the other claustrum contained a much smaller region of tracer overlap in which anterograde and retrograde labeling was less intense.

Bilateral tracer injections in the Re regions of MI produced asymmetric patterns of claustral labeling that resembled the hemispheric patterns produced by the MI-RW injections, but the overall intensity and spatial extent of the labeling was reduced. An injection of retrograde tracer into the MI-Re region of the right hemisphere revealed dozens of labeled neurons in the right claustrum but very few neurons in the left claustrum (Fig. $3 A-H)$. An injection of anterograde tracer into the left MI-Re region of the same rat produced moderately dense terminal labeling in the right claustrum, but the labeling in the left claustrum was relatively sparse. Consequently, when compared with the MI-RW injections, the bilateral tracer injections in the MI-Re regions produced a small region of tracer overlap in the claustrum located ipsilateral to the retrograde tracer injection but very little tracer overlap in the other claustrum.

Tracer injections in the MI-Fp regions produced almost no claustral labeling. As shown in Figure 3I-P, very few anterogradely labeled terminals or retrogradely labeled soma were observed in the claustrum of either hemisphere. A few labeled varicosities were scattered in the claustral region where the dorsal and ventral subnuclei adjoin each other, but the density was too low to produce noticeable labeling in photomicrographs obtained at low magnification (Fig. 3M--P). Labeled neurons were virtually absent from the claustrum located contralateral to the retrograde tracer (FG) injection (Fig. $3 J, N$ ), and only a sparse group of labeled soma appeared in the ventral and dorsal subnuclei on the ipsilateral side (Fig. $3 K, O$ ). Hence, because tracer injections in the MI-Fp regions produced very little claustral labeling, tracer overlap was not apparent in the claustrum of either hemisphere.

Statistical analysis confirmed that the number of retrogradely labeled neurons in the claustrum was affected by the functional locations (RW, Re, Fp) of the FG injections in MI $(F=15.7$; $p<$ $0.0001)$. Furthermore, the hemispheric location (contralateral or ipsilateral) of the claustrum had a significant effect on the number of retrogradely labeled neurons $(F=68.2 ; p<0.0001)$. When the number of labeled claustral neurons on each side was compared section by section (11 sections analyzed in each rat), a matched-sample analysis showed that neuronal labeling was significantly higher in the hemisphere that received the retrograde tracer, regardless of whether the tracer was placed in the RW (paired $t=6.5 ; p<0.000001$ ) or $\operatorname{Re}($ paired $t=5.4 ; p<0.00001$ ) regions of $\mathrm{MI}$. 

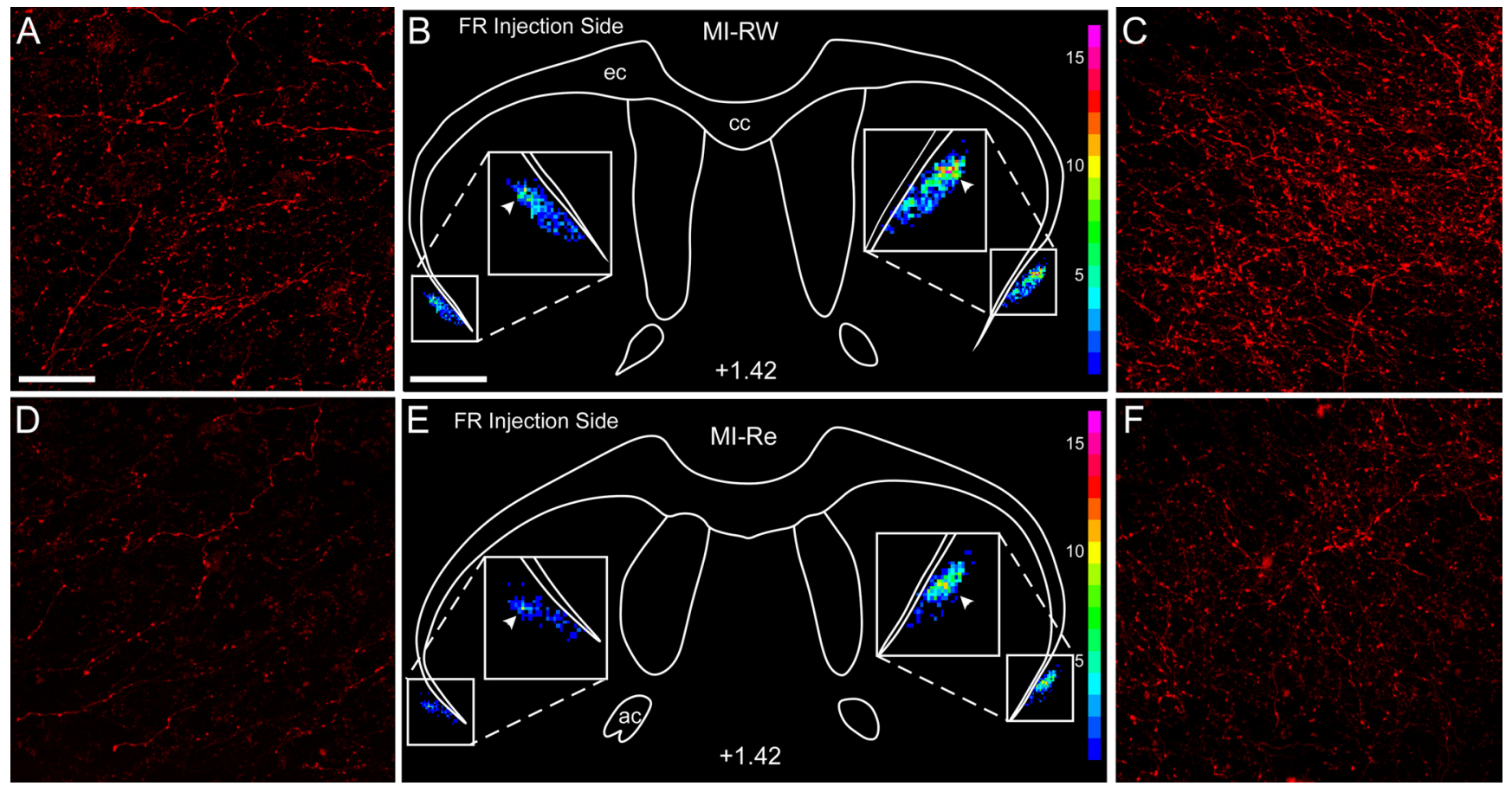

Figure 5. Relative density of corticoclaustral projections from FR injection sites in the MI-RW (CL21) and MI-Re (CL05) regions. A, C, Confocal images of FR-labeled varicosities in the claustrum of the ipsilateral and contralateral hemispheres after an FR injection into the RW region. $\boldsymbol{B}$, Color-coded reconstructions of the density of plotted varicosities in the claustrum of each hemisphere. The number of plotted varicosities in each $25 \mu \mathrm{m}^{2}$ bin is indicated by the color scale on the right. Inset, Arrowheads indicate the region from which the confocal images in $\boldsymbol{A}$ and $\boldsymbol{C}$ were obtained. $\boldsymbol{D}-\boldsymbol{F}$, Density of FR-labeled varicosities after injecting the tracer into the MI-Re region; images and reconstructions are presented as in $\boldsymbol{A}$ and $\boldsymbol{C}$. Scale bars: $\boldsymbol{A}, \boldsymbol{C}-\boldsymbol{E}, 25 \mu \mathrm{m} ; \boldsymbol{B}, \boldsymbol{E}, 1 \mathrm{~mm}$.

Similar analyses confirmed that the number of anterogradely labeled varicosities in the claustrum was affected by the MI location of the FR tracer injections $(F=51.1 ; p<0.0001)$ and by the hemispheric location of the claustrum $(F=22.7 ; p<0.0001)$. A matched-sample section-by-section comparison demonstrated that the number of labeled varicosities was significantly higher in the contralateral claustrum when the anterograde tracer was injected into the RW (paired $t=6.3 ; p<0.000001$ ) or Re (paired $t=5.6$; $p<0.00001$ ) regions of MI.

\section{Relative strength of claustrum connections with the MI whisker regions}

For the six rats in which tracers were injected into the MI whisker regions (RW or Re), asymmetries in the relative balance of labeling across the two hemispheres were apparent in all claustral sections that contained labeling. As shown in Figure 4, regardless of which MI whisker region was injected, labeled neurons were mainly in the claustrum located ipsilateral to the retrograde tracer injection (Fig. 4A). Conversely, labeled terminals were consistently more numerous in the claustrum located contralateral to the anterograde tracer injection (Fig. $4 B$ ).

Although tracer injections in both MI whisker regions produced similar labeling patterns, several observations indicate that the claustrum is more strongly connected with the RW than with the Re region. At each rostrocaudal position (Fig. $4 \mathrm{~A}$ ), retrograde tracer injections in MI-RW revealed more labeled neurons in the ipsilateral claustrum than similar tracer injections in the MI-Re region. Statistical analysis confirmed that these differences were significant $(t=2.4 ; p<0.05)$. Furthermore, despite the fact that exactly $100 \mathrm{nl}$ of the anterograde tracer (FR) was pressure injected in each rat, the total number of labeled varicosities in the claustrum of both hemispheres was highest when the tracer was injected into the MI-RW region $(t=4.0 ; p<0.001)$. When each

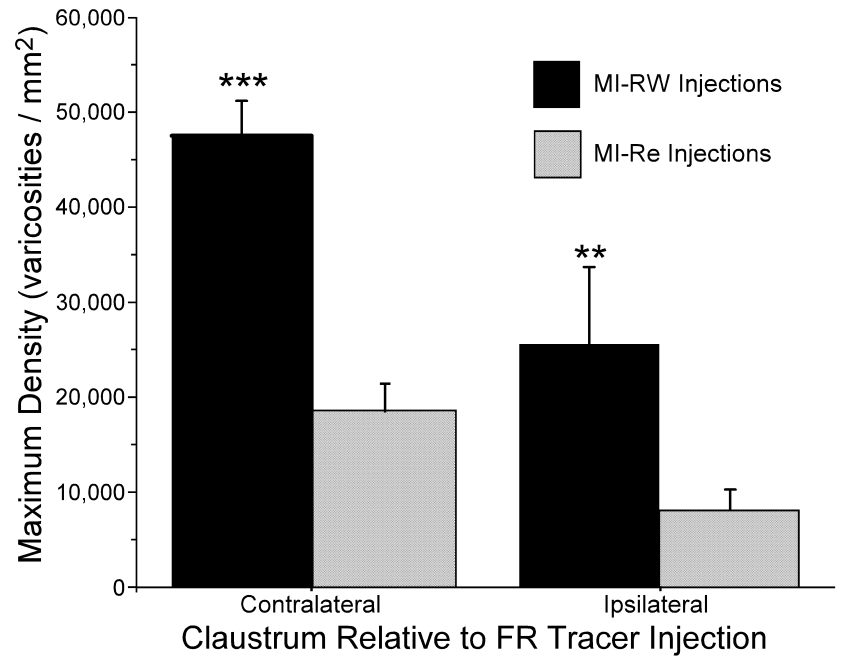

Figure 6. Bar graphs depicting the maximum density of labeled varicosities in the claustrum after FR injections into the RW or Re regions. Labeled varicosities were counted from confocal images obtained from claustral regions with the most FR labeling. Each bar represents the mean varicosity density calculated from nine confocal images obtained from three rats. Error bars represent SEM; asterisks indicate that RW injections produced a significant increase in the maximum density of FR labeling $\left({ }^{* *} p<0.01 ;{ }^{* * *} p<0.001\right)$.

hemisphere was analyzed separately, anterograde tracer injections in the RW region produced more labeled varicosities in the claustrum than similar injections in the Re region for both the ipsilateral $(t=2.9 ; p<0.01)$ and contralateral $(t=4.1 ; p<$ $0.001)$ hemispheres.

We also analyzed the areal extent of terminal labeling in the claustrum after anterograde tracer injections in the RW and Re regions. For this purpose, the plotted reconstructions of the 

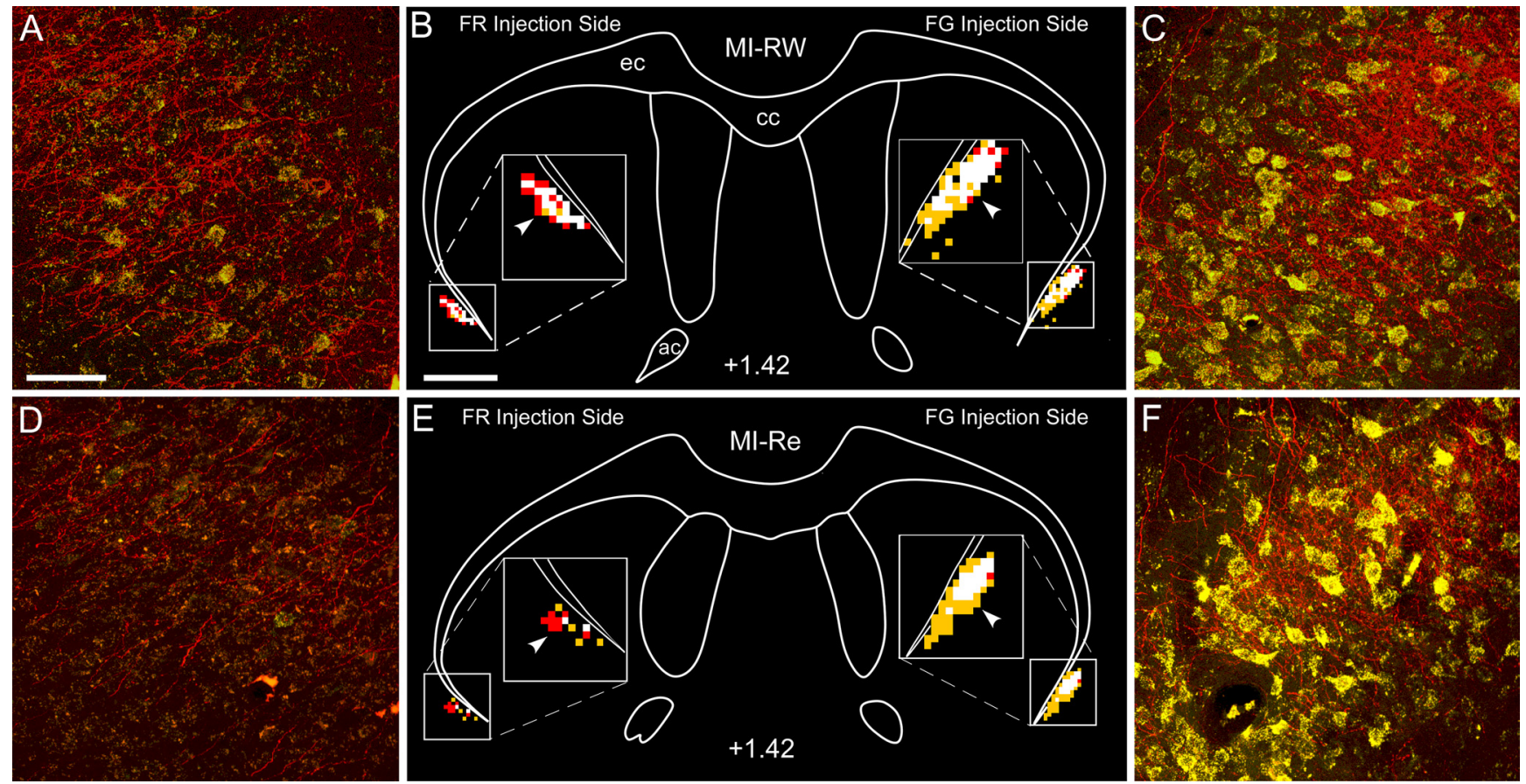

Figure 7. Bilateral distribution of tracer overlap in the claustrum after injections of FR and FG into MI-RW and MI-Re. $A, C$, Confocal images of FR-labeled varicosities and FG-labeled neurons in the claustrum of the left and right hemispheres after tracer injections in the RW region. $\boldsymbol{B}$, Color-coded reconstruction of tracer overlap in the MI-RW case. Bins $\left(50 \mu \mathrm{m}^{2}\right)$ colored red contain four or more FR-labeled varicosities, yellow bins contain one or more FG-labeled neurons, and white bins contain four or more FR-labeled varicosities with one or more FG-labeled neurons. $\mathbf{D}-\boldsymbol{F}$, Tracer overlap produced by tracer injections in the MI-Re regions; images and reconstruction are depicted as in $\boldsymbol{A}-\boldsymbol{C}$. Arrowheads $(\boldsymbol{B}, \boldsymbol{E})$ indicate claustral regions from which confocal images were obtained. Scale bars: $A, C, D, F, 50 \mu \mathrm{m} ; \boldsymbol{B}, \boldsymbol{E}, 1 \mathrm{~mm}$. ec, External capsule; ac, anterior commissure; cc, corpus callosum.

claustral labeling patterns were subdivided into a grid of $25 \mu \mathrm{m}^{2}$ bins, and variations in the number of plotted varicosities in each bin were represented by a color-coded map of labeling density. As shown by these color-coded reconstructions in Figure 5, projections from the RW region innervated a larger claustral area than the projections from the Re region. Analysis of the spatial extent of anterograde labeling in the claustrum, as measured by the sum of the colored bins $\left(25 \mu \mathrm{m}^{2}\right.$ each) across all sections, indicated that the RW region innervated a larger portion of the claustrum than the Re region $(t=2.0 ; p<0.05)$. This difference was mainly caused by the increased innervation of the contralateral claustrum $(t=2.0 ; p<0.05)$, as the innervated area in the ipsilateral claustrum was not significantly different for anterograde tracer injections in the RW or Re regions $(t=1.8 ; p=0.07)$.

The color-coded reconstructions indicated variations in the density of anterogradely labeled varicosities across different parts of the claustrum. As indicated in Figure 5, $B$ and $E$, labeled varicosities were densest in the dorsal half of the labeled area. The high density of labeling in this region, however, made it impossible to plot each individual varicosity using conventional fluorescent microscopy. To obtain an accurate count of the labeled varicosities in these densely labeled regions, confocal images were acquired from the sites where our colorcoded reconstructions indicated that anterograde labeling was densest (Fig. $5 B, E$, arrowheads). Consistent with the rostrocaudal distribution of terminal labeling (Fig. 4B), confocal images were obtained from three coronal sections located $0.70,1.40$, and $2.10 \mathrm{~mm}$ rostral to bregma. For each section, a confocal image was obtained from both hemispheres as indicated in Figure 5. The number of labeled varicosities in each image was counted by two individuals who were unaware of the image identity, and these counts were averaged for each image (see Materials and Methods).
Visual inspection of the confocal images in Figure 5 suggests that the maximum density of labeled varicosities was highest when the anterograde tracer was placed in the MI-RW region. In fact, statistical analysis confirmed that the density of labeled varicosities was significantly higher when the anterograde tracer was injected in the MI-RW region. As indicated in Figure 6, differences in the maximum density of labeled varicosities produced by tracer injections in the RW and Re regions were significant for both the contralateral $(t=5.1 ; p<0.001)$ and ipsilateral $(t=3.4$; $p<0.01)$ hemispheres.

\section{Interhemispheric cortico-claustro-cortical circuits}

Simultaneous visualization of both tracers indicated that a large portion of the anterogradely labeled terminals were intermingled with retrogradely labeled neurons when the tracers were injected into MI-RW or MI-Re. In fact, as shown by the confocal images in Figure 7, $C$ and $F$, the high density of labeled terminals prevented complete visualization of many of the labeled cell bodies. Although ultrastructural evidence is needed to confirm synaptic connectivity, the dense intermingling of the anterogradely labeled terminals and retrogradely labeled neurons indicates that an interhemispheric cortico-claustral-cortical circuit could be mediated by monosynaptic connections in the claustrum.

To assess potential differences in the amount of tracer overlap produced by tracer injections in the RW or Re regions, the plotted reconstructions of the two-tracer labeling patterns were subdivided into $50 \mu \mathrm{m}^{2}$ bins. Compared with our spatial analysis of labeled terminals, which was based on grids containing $25 \mu \mathrm{m}^{2}$ bins (Fig. 5), spatial analysis of tracer overlap was initially based on grids containing larger bins because neurons are much larger than axonal varicosities. Furthermore, because multiple varicosities are more likely to produce postsynaptic effects, at least four or more labeled varicosities had to reside in a bin with a labeled 
neuron to represent a bin with overlapping tracers. Therefore, as shown in Figure 7, $B$ and $E$, bins in which we plotted at least one retrogradely labeled neuron and four or more anterogradely labeled varicosities were colored white. In comparison, bins with four or more FR-labeled varicosities but no FG-labeled neurons were colored red, whereas bins that contained at least one FGlabeled neuron but fewer than four FR-labeled varicosities were colored yellow. All of the remaining bins were colored black. The white bins in each hemisphere were counted to provide an index of the spatial extent of terminal-soma overlap in the two groups (RW and $\mathrm{Re}$ ) of rats that received tracer injections in the MI whisker regions.

As shown in Figures 7 and 8, this methodology revealed clear hemispheric differences in the amount of tracer overlap. Consistent with the fact that the vast majority of labeled neurons were located ipsilateral to the retrograde tracer injection, the areal extent of tracer overlap (i.e., number of white bins) was always highest in the claustrum located in the hemisphere that received the retrograde tracer. This hemispheric difference in tracer overlap was significant regardless of whether the tracers were injected into the RW (paired $t=6.16 ; p<0.001$ ) or Re (paired $t=3.97$; $p<0.01)$ regions.

Furthermore, tracer injections in MI-RW produced more overlap in the claustrum than injections in MI-Re. As indicated by the reconstructions in Figure 7, tracer overlap was more extensive in the RW than in the Re case. When the white bins were summed across both hemispheres, statistical analysis confirmed that total tracer overlap in the RW cases was significantly greater than the overlap produced by injections in the $\mathrm{Re}$ regions $(t=2.9 ; p<0.01)$. In fact, tracer overlap was significantly higher for the RW cases regardless of whether the analysis was applied to the hemisphere that received the retrograde tracer $(t=2.8 ; p<$ $0.01)$ or the one that received the anterograde tracer $(t=2.6 ; p<0.05)$.

The difference in tracer overlap between the RW and Re cases did not depend on bin size or the threshold number of labeled varicosities in each bin. Although the absolute amount of tracer overlap varied with bin size $(25,50$, or 100 $\mu \mathrm{m}^{2}$ ) and with the threshold number of labeled varicosities required for each bin (one, two, four, or six), the relative extent of tracer overlap was always higher for rats in which both tracers were injected into the RW regions (data not shown).

\section{Retrograde labeling of corticoclaustral projection neurons}

In a separate set of rats, the retrograde tracer (FG) was placed in the claustrum or surrounding regions. These injections were targeted at the rostrocaudal levels of the claustrum $(1.0-1.5 \mathrm{~mm}$ rostral to bregma) that received the greatest number of projections from the MI whisker region (Fig. 4). Among 11 rats that received tracer injections aimed at the claustrum, tracer deposits in 9 rats were either completely outside the claustrum or involved both the claustrum and extensive parts of the surrounding regions. When the tracer was injected entirely outside the claustrum $(n=6)$, either into the insular cortex or into the ventrolateral striatum, very few retrogradely labeled neurons ap-

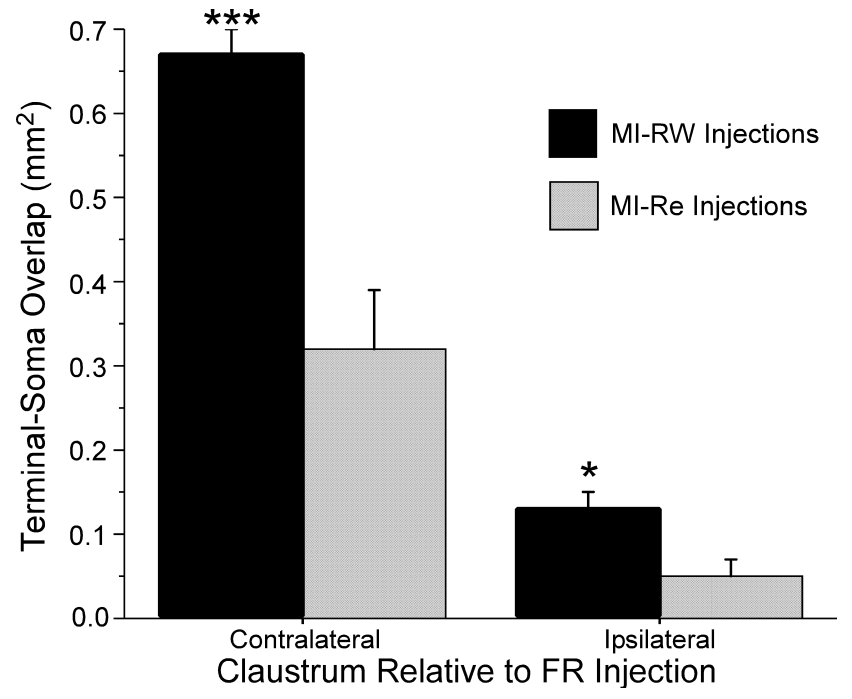

Figure 8. Bar graphs depicting the spatial extent of terminal-soma overlap in the claustrum after tracer injections in the RW or Re regions. The overlap area was calculated from the number of $50 \mu \mathrm{m}^{2}$ bins that were colored white, as shown in Figure 7. Each bar represents the mean area of tracer overlap in three rats. Error bars represent SEM; asterisks indicate that RW injections produced significantly more overlap than Re injections $\left({ }^{*} p<0.05 ;{ }^{* * *} p<0.001\right)$.
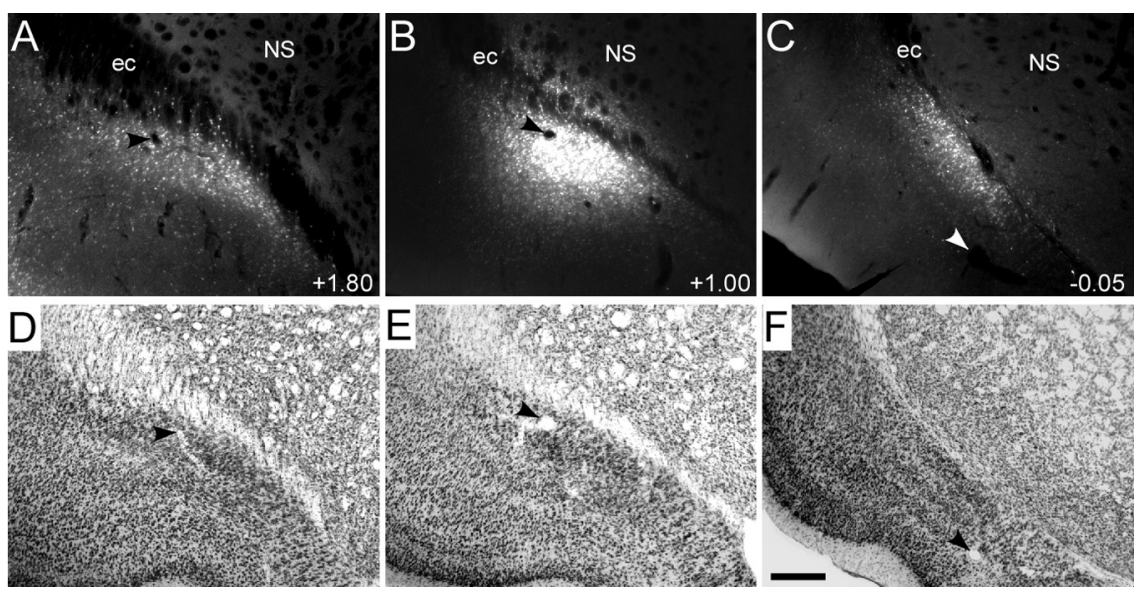

Figure 9. Intraclaustral labeling patterns produced by a focal tracer injection in the claustrum. $\boldsymbol{A}, \boldsymbol{C}, \operatorname{Rostral}(\boldsymbol{A})$ and caudal ( $\boldsymbol{C})$ the FG injection in the claustrum. $\boldsymbol{D}-\boldsymbol{F}$, Adjacent sections illustrating cytoarchitecture of the claustrum at each rostrocaudal leve depicted in A-C. Numbers (bottom right) indicate rostrocaudal distance from bregma; arrowheads indicate same blood vessels in adjacent sections. Scale bar, $250 \mu \mathrm{m}$ for all panels. ec, External capsule; NS, neostriatum.

peared in MI cortex. This result is consistent with the fact that our anterograde tracer injections in the RW and Re regions produced terminal labeling in the claustrum but not in the insular cortex or ventral striatum.

Injecting the claustrum alone proved difficult, and only two rats received retrograde tracer deposits that were largely restricted to the claustrum. In the case shown in Figure 9, the tracer filled most of the ventral claustrum, a small portion of the dorsal claustrum, and possibly the surrounding edges of the insular cortex. The other case involved tracer that was mainly in the dorsal claustrum, the upper part of the ventral claustrum, and in the lateral edge of the ventral striatum. These retrograde tracer deposits were relatively compact, and histologic inspections indicated that the tracer diffused $<400 \mu \mathrm{m}$ in the rostral and caudal directions.

In the cases in which the FG was placed almost entirely in the 

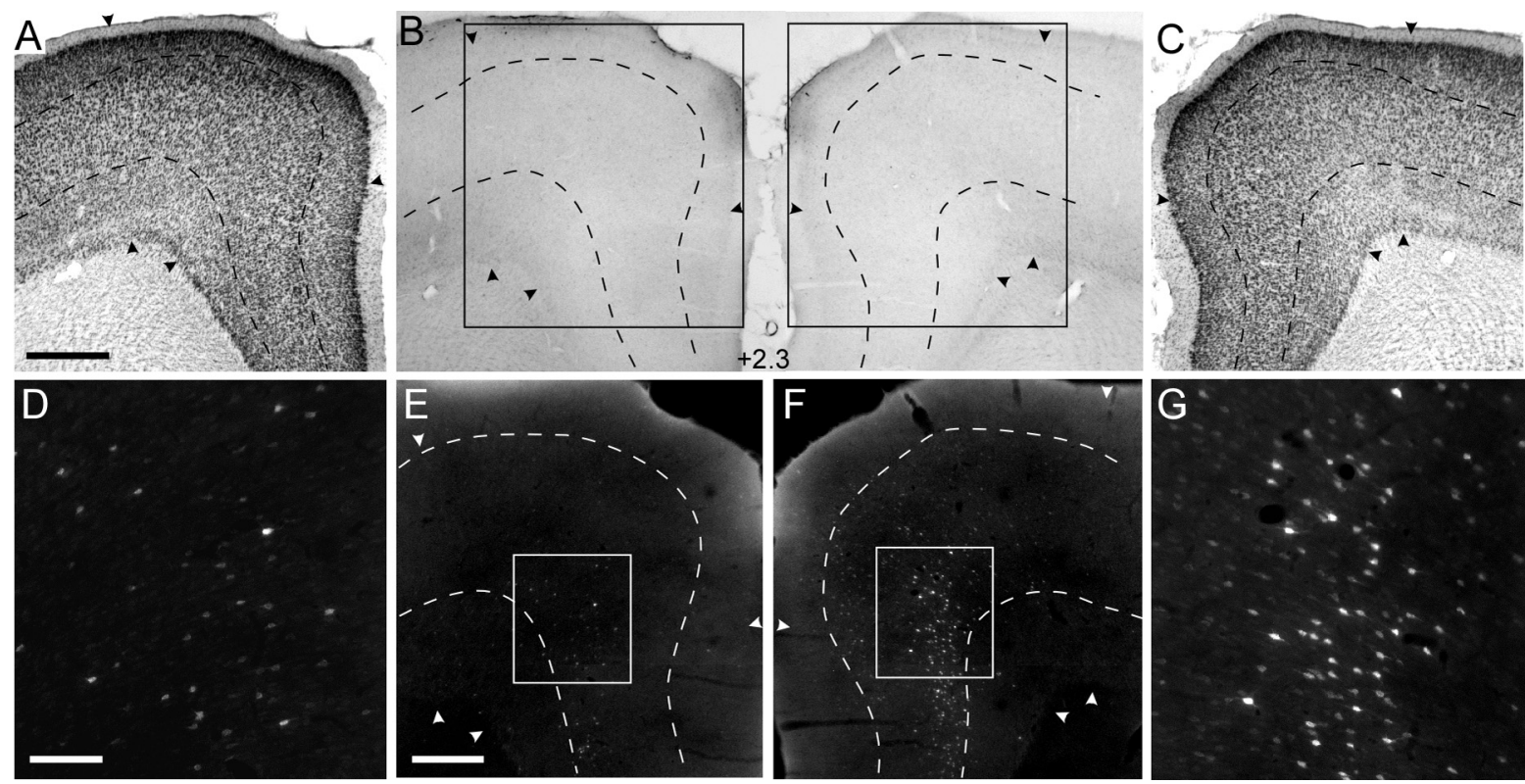

Figure 10. Labeled neurons in MI cortex produced by the tracer injection in the left claustrum as shown in Figure 9.A, C, Thionin-stained sections show the cytoarchitecture of area Agm in the left and right hemispheres, respectively. Arrowheads indicate cytotectonic borders of Agm cortex. $\boldsymbol{B}$, Adjacent unstained sections used for fluorescent microscopy. Rectangles indicate the regions in $\boldsymbol{E}$ and $\boldsymbol{F}$; the number indicates the distance from bregma. $\boldsymbol{D}, \boldsymbol{G}$, Magnified views of labeled neurons in Agm cortex of the left and right hemispheres, respectively. $\boldsymbol{E}, \boldsymbol{F}$, Low-power views of neuronal labeling with respect to the borders of Agm cortex (arrowheads) and layer $V$ (dashed line). Rectangles indicate regions depicted in $\boldsymbol{D}$ and $\mathbf{G}$. Scale bars: $\boldsymbol{A}-\boldsymbol{C}, 500 \mu \mathrm{m} ; \boldsymbol{D}, \boldsymbol{G}, 100 \mu \mathrm{m} ; \boldsymbol{E}, \boldsymbol{F}, 250 \mu \mathrm{m}$.

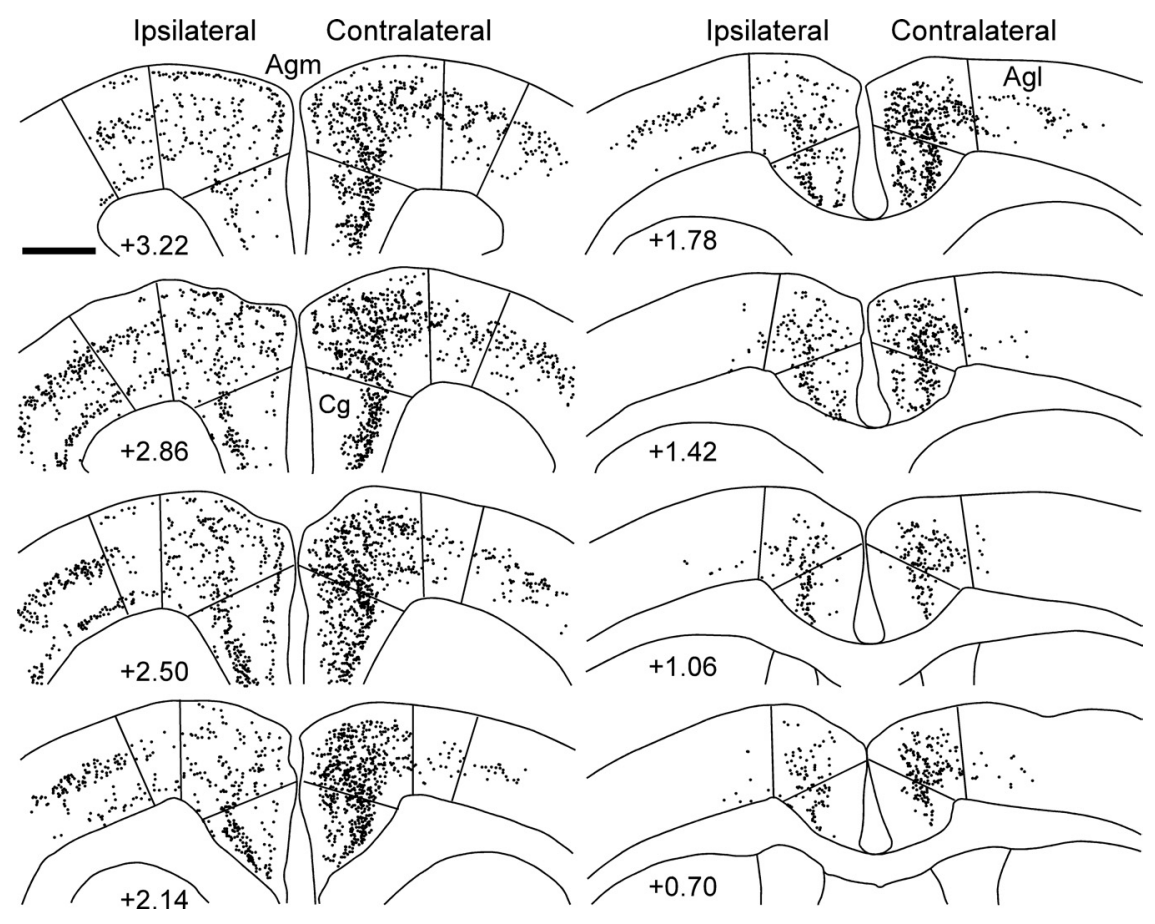

Figure 11. Reconstructions illustrating the distribution of retrogradely labeled cortical neurons after focal injection of FG into the left claustrum as shown in Figure 9. Each dot represents the location of an FG-labeled neuron. Numbers (bottom left) indicate distance from bregma; lines indicate borders between cortical areas $\mathrm{Cg}$, Agm, and Agl determined from the cytoarchitecture of adjacent thionin-stained sections. Scale bar, $1 \mathrm{~mm}$.

claustrum, hundreds of retrogradely labeled neurons appeared throughout elongated portions of the ipsilateral claustrum (Fig. $9 A, C)$. Neuronal labeling did not appear in the claustrum, however, when large deposits of the retrograde tracer were injected in the insular cortex or in the ventral striatum (data not shown). Hence, these collective results indicate extensive longitudinal axonal projections within the claustrum itself.
Injections of FG into the claustrum revealed large numbers of retrogradely labeled cortical neurons in Cg, Agm, and Agl cortices and related frontal areas, but not in other cortical areas. Despite suggestions that the claustrum integrates multimodal information received from different cortical areas (Edelstein and Denaro, 2004; Crick and Koch, 2005), our tracer injections in the claustrum failed to produce neuronal labeling in somatosensory barrel cortex or other somatosensory regions such as the secondary somatosensory cortex or the parietal ventral area. Nor did any labeling appear in more caudal regions that contain visual or other sensory-processing areas. The total lack of labeling in these nonmotor cortical areas is consistent with physiological data in the primate showing that local regions in the claustrum are modality specific and do not respond to multimodal inputs (Remedios et al., 2010).

As shown in Figures 10 and 11, the cortical labeling produced by retrograde tracer injections in the claustrum was greatest in the Agm and Cg cortices of the contralateral hemisphere. A section-bysection analysis indicated that the number of labeled neurons was significantly higher in the contralateral than in the ipsilateral hemisphere for both the Agm (paired $t=4.1 ; p<0.01$ ) and $\mathrm{Cg}$ (paired $t=2.6 ; p=0.05$ ) cortical areas. We did not detect any hemispheric differences among the smaller number of labeled neurons in Agl cortex (paired $t=0.8 ; p=0.4$ ).

The neuronal labeling in Agm of the contralateral hemisphere was extremely dense in the medial part of this region that adjoins 
the Cg cortex. Photomicrographs of this densely labeled site illustrate high concentrations of brightly labeled neurons in layer $\mathrm{V}$ near the border separating the medial edge of Agm cortex from the underlying Cg cortex (Fig. 10F, G). The plotted reconstructions also indicate that the density of neuronal labeling was highest at this site (Fig. 11). To portray more precisely the spatial variations in the density of neuronal labeling, adjacent pairs of these reconstructions were superimposed and subdivided into a grid of $100 \mu \mathrm{m}^{2}$ bins that were color coded according to the number of labeled neurons in each bin. As indicated in Figure 12, this procedure indicated that neuronal labeling was densest at the medial edge of Agm cortex that borders the Cg cortex. This focal region of dense labeling was apparent at the same location in all sections obtained $1.0-2.5 \mathrm{~mm}$ rostral to bregma, a region that corresponds to the same MI area that we and others have microstimulated to evoke rhythmic whisking movements (Haiss and Schwarz, 2005).

Changes in the laminar pattern of neuronal labeling were apparent at the cytoarchitectonic transitions between Agm cortex and its neighboring cortical areas. In contrast to Agm cortex, in which labeled neurons appeared in layer $\mathrm{V}$ and more superficial layers, the density of neuronal labeling dropped sharply at the border between Agm and Agl cortices. Interestingly, neuronal labeling in Agl cortex had a distinct laminar pattern in the rostral parts of the ipsilateral hemisphere but showed less laminar organization in the contralateral hemisphere.

In both hemispheres, the border between Agm and Cg cortices was marked by a distinct change in the laminar organization of neuronal labeling. In contrast to Agm cortex, in which labeled neurons appeared in layer $\mathrm{V}$ and more superficially, most labeled neurons in Cg cortex were concentrated in layer V. Layer V is relatively thick in Agm cortex but becomes substantially thinner in Cg cortex. Consequently, because layer III in Cg cortex contains few projections to the claustrum, the high density of layer $\mathrm{V}$ labeling in both areas accentuates the border between them as this layer becomes compressed in the transition from Agm to $\mathrm{Cg}$ cortex.

\section{Discussion}

Our bilateral tracer injections revealed an interhemispheric cortico-claustro-cortical circuit that connects corresponding parts of MI cortex. This interhemispheric circuit connects MI regions associated with whisker retractions or rhythmic whisker movements, but not the MI regions linked to forepaw movements. Quantitative analysis indicates that interhemispheric connections between the MI-RW regions are stronger than the connections between the MI-Re regions. This finding was confirmed by retrograde tracer injections in the claustrum showing that the densest neuronal labeling was in the medial part of the contralateral Agm cortex that represents the MI-RW region. Furthermore, the tracer injections in the claustrum revealed extensive interconnections along its rostrocaudal length.

\section{Cortico-claustro-cortical circuit connections}

We previously used anterograde tracers to demonstrate that the MI whisker region projects bilaterally to the claustrum, but with more numerous projections to the contralateral than to the ipsilateral side (Alloway et al., 2009). In another report (Colechio and Alloway, 2009), injections of retrograde tracers in MI established that the vast majority of claustral projections to the MI whisker region originate from the ipsilateral side. The present study used bilateral combinations of both types of tracers to demonstrate that corticoclaustral projections to the contralateral hemisphere terminate in regions that overlap the neuronal soma that form the origin of the claustrocortical pathway in that hemisphere. Indeed, the high density of terminal and neuronal labeling in overlapping regions makes it highly probable that monosynaptic connections in the claustrum convey information from the contralateral MI cortex to the corresponding motor region in the ipsilateral hemisphere.

Previous work indicates that MI cortex projects bilaterally to several structures that could coordinate the motor cortical areas in both hemispheres. Several studies, for example, demonstrate that MI cortex projects bilaterally to the striatum (Wilson, 1986, 1987; Reiner et al., 2003; Reep et al., 2008; Alloway et al., 2009). The MI whisker region also projects bilaterally to the thalamus, especially the ventrolateral, ventromedial, and intralaminar nuclei (Molinari et al., 1985; Rouiller et al., 1991; Alloway et al., 2008).

The MI projections to the claustrum, however, are different from the MI projections to the thalamus and striatum. Although the MI whisker region projects more strongly to the contralateral claustrum, corticothalamic and corticostriatal projections from MI cortex terminate predominantly in the ipsilateral hemisphere [Alloway et al., their Fig. 9A (2008) and Fig. 12 (2009)]. In addition, corticoclaustral projections to the contralateral hemisphere have a higher density of terminal varicosities than MI projections to the contralateral striatum or thalamus [Alloway et al. (2009), their Fig. 7]. This is consistent with the fact that MI whisker projections to the claustrum are concentrated in a limited territory, whereas corticothalamic and corticostriatal projections are less dense and innervate much larger regions.

Other aspects of the cortico-claustro-cortical circuit distinguish it from the interhemispheric circuits that involve the striatum or thalamus. Information processed in the striatum is conveyed across multiple synapses in the basal ganglia and thalamus before it reaches MI cortex. Hence, the basal gangliathalamocortical loop is longer and, undoubtedly, slower than the claustral circuit in coordinating MI regions in both hemispheres. 
Furthermore, the MI-Fp region sends few projections to the claustrum in either hemisphere but projects strongly to the ipsilateral thalamus and to the striatum in both hemispheres (Alloway et al., 2009). Hence, the connectional data suggest important functional distinctions in the content of information and its speed of transmission through the cortico-claustral-cortical circuit and other interhemispheric circuits originating from MI.

\section{Intraclaustral connections}

Whereas many anatomical studies have revealed similarities in the afferent and efferent connections of the claustrum in a number of mammalian species (for review, see Sherk, 1986; Edelstein and Denaro, 2004), there is little information regarding neuronal connectivity within the claustrum. The claustrum contains a limited number of neuronal cell types, and several studies have characterized claustral neurons according to their size, shape, and neurochemical features (Eiden et al., 1990; Druga et al., 1993; Kowianski et al., 2001, 2008). Yet, no study has characterized the axonal processes of claustral neurons to determine the extent of intraclaustral connectivity. The present study, however, established extensive interconnections along the claustrum's rostrocaudal axis. Placement of a retrograde tracer in the claustrum revealed hundreds of labeled neurons throughout its longitudinal extent, but the surrounding cortex was devoid of labeled neurons. When large retrograde tracer injections were placed in the insular cortex adjacent to the claustrum, we observed labeled neurons in the cortical regions that surround the claustrum throughout its rostrocaudal extent but saw very few labeled neurons in the claustrum. Hence, the extensive claustral labeling observed after a retrograde tracer injection in the claustrum must be attributable to long-range intraclaustral connections that were labeled by the bulk of the tracer deposit in the claustrum and not by the small quantity of tracer that may have diffused into the surrounding cortex.

These long-range connections indicate that information travels widely throughout the claustrum. The precise nature of this information is not known, however, and our data do not indicate whether these long claustral projections represent communication between separate parts of the same motor-based claustral region or between parts of the claustrum that process different cortical modalities. Nonetheless, intraclaustral connections could provide a mechanism for binding near-coincident inputs from cortical areas that process related aspects of the same behavioral activity (Crick and Koch, 2005).

The presence of extensive intraclaustral connections, coupled with the convergence of inputs from large parts of Cg and Agm cortices, has important implications for the transmission of information through the claustrum and its impact on postsynaptic cortical targets. Regardless of whether the intraclaustral connections represent the axons of interneurons or the collaterals of claustrocortical projections, either type of connection could synchronize extensive parts of the claustrum. This view is consistent with data indicating that the claustrum is susceptible to kindling and plays a role in the development of epileptiform activity (Mohapel et al., 2000, 2001). If many claustral neurons converge on overlapping cortical regions, synchronization of these neurons should effectively activate their cortical targets. In this context, it is worth noting that our relatively small injections of retrograde tracers in the MI-RW region produced widespread neuronal labeling throughout the rostrocaudal extent of the claustrum. Hence, the high density of overlapping afferent (FRlabeled) and efferent (FG-labeled) claustral connections, along with the its extensive intrinsic connectivity and the likelihood of neuronal synchronization, demonstrates that the claustrum has connectional properties that should optimize the transmission of information from one MI region to its counterpart in the other hemisphere.

\section{Neural and behavioral functions of interhemispheric claustral circuits}

The MI whisker regions in each hemisphere are directly interconnected by dense sets of axons that project through the corpus callosum (Donoghue and Parham, 1983; Reep et al., 1987; Miyashita et al., 1994). The afferent and efferent connections of the claustrum provide another route, probably monosynaptic, that complements the direct callosal connections between the MI whisker regions in each hemisphere. By including an intervening synapse along this second route, the claustrum offers an opportunity for integrating cortical information from the ipsilateral hemisphere with information received from the MI cortex in the contralateral hemisphere. The claustrum projects to both the Re and RW regions in MI, and this could coordinate their interactions during whisking behavior. Depending on the details of the synaptic organization in both the claustrum and MI cortex, the information transmitted by this interhemispheric circuit could reinforce the bilateral symmetry whisker movements or, alternatively, decouple the whisker movements in response to head movements or other sensory inputs (Towal and Hartmann, 2006; Mitchinson et al., 2007). Determining the specific actions mediated by claustral circuits must await future research that examines the behavioral effects of manipulating the claustrum.

From a systems perspective, the functional significance of the interhemispheric claustral circuit is inferred from the fact it is structured to coordinate MI cortical areas that regulate eye and whisker movements (i.e., $\mathrm{Cg}$ and Agm), but not the MI areas associated with forelimb movements (i.e., Agl). Although forelimb movements are bilaterally coordinated to support the body and move it through space, the eyes and whiskers are bilaterally coordinated to acquire sensory information for perceptual awareness of a wide portion of the surrounding environment, especially as it relates to spatial orientation and directed attention. In contrast to forelimb movements, which have a limited ability for quickly sampling multiple stimuli, bilateral whisking movements can rapidly detect nearby stimuli throughout the three-dimensional space surrounding the head. Consistent with this, the MI whisker regions receive inputs from the posterior parietal cortex (PPC), but the MI forepaw region does not (Colechio and Alloway, 2009). The PPC processes multimodal sensory inputs that are needed to modulate $\mathrm{MI}$ and guide motor output for spatial orientation and directed attention (Dijkerman and de Haan, 2007; Reep and Corwin, 2009). Hence, the interhemispheric claustral circuit described in the present study probably coordinates the motor cortical regions that regulate movements involved in the acquisition of sensory information for global, largescale perception.

\section{References}

Alloway KD, Olson ML, Smith JB (2008) Contralateral corticothalamic projections from MI whisker cortex: potential route for modulating hemispheric interactions. J Comp Neurol 510:100-116.

Alloway KD, Smith JB, Beauchemin KJ, Olson ML (2009) Bilateral projections from rat MI whisker cortex to the neostriatum, thalamus, and claustrum: forebrain circuits for modulating whisking behavior. J Comp Neurol 515:548-564.

Alloway KD, Smith JB, Beauchemin KJ (2010) Quantitative analysis of the bilateral brainstem projections from the whisker and forepaw regions in rat primary motor cortex. J Comp Neurol 518:4546-4566. 
Brecht M, Krauss A, Muhammad S, Sinai-Esfahani L, Bellanca S, Margrie TW (2004) Organization of rat vibrissa motor cortex and adjacent areas according to cytoarchitectonics, microstimulation, and intracellular stimulation of identified cells. J Comp Neurol 479:360-373.

Carey RG, Neal TL (1985) The rat claustrum: afferent and efferent connections with visual cortex. Brain Res 329:185-193.

Clarey JC, Irvine DR (1986) Auditory response properties of neurons in the claustrum and putamen of the cat. Exp Brain Res 61:432-437.

Colechio EM, Alloway KD (2009) Differential topography of the bilateral cortical projections to the whisker and forepaw regions in rat motor cortex. Brain Struct Funct 213:423-439.

Cortimiglia R, Crescimanno G, Salerno MT, Amato G (1991) The role of the claustrum in the bilateral control of frontal oculomotor neurons in the cat. Exp Brain Res 84:471-477.

Crick FC, Koch C (2005) What is the function of the claustrum? Philos Trans R Soc Lond B Biol Sci 360:1271-1279.

Dijkerman HC, de Haan EH (2007) Somatosensory processes subserving perception and action. Behav Brain Sci 30:189-239.

Donoghue JP, Parham C (1983) Afferent connections of the lateral agranular field of the rat motor cortex. J Comp Neurol 217:390-404.

Druga R, Chen S, Bentivoglio M (1993) Parvalbumin and calbindin in the rat claustrum: an immunocytochemical study combined with retrograde tracing frontoparietal cortex. J Chem Neuroanat 6:399-406.

Edelstein LR, Denaro FJ (2004) The claustrum: a historical review of its anatomy, physiology, cytochemistry and functional significance. Cell Mol Biol 50:675-702.

Eiden LE, Mezey E, Eskay RL, Beinfeld MC, Palkovits M (1990) Neuropeptide content and connectivity of the rat claustrum. Brain Res 523:245250.

Haiss F, Schwarz C (2005) Spatial segregation of different modes of movement control in the whisker representation of rat primary motor cortex. J Neurosci 25:1579-1587.

Hall RD, Lindholm LE (1974) Organization of motor and somatosensory neocortex in the albino rat. Brain Res 66:23-38.

Hoffer ZS, Hoover JE, Alloway KD (2003) Sensorimotor corticocortical projections from rat barrel cortex have an anisotropic organization that facilitates integration of inputs from whiskers in the same row. J Comp Neurol 466:525-544.

Kincaid AE, Wilson CJ (1996) Corticostriatal innervation of the patch and matrix in the rat neostriatum. J Comp Neurol 374:578-592.

Kowianski P, Dziewiatkowski J, Kowianska J, Morys J (1999) Comparative anatomy of the claustrum in selected species: a morphometric analysis. Brain Behav Evol 53:44-54.

Kowianski P, Timmermans J-P, Morys J (2001) Differentiation in the immunocytochemical features of intrinsic and cortically projecting neurons in the rat claustrum - combined immunocytochemical and axonal transport study. Brain Res 905:63-71.

Kowianski P, Morys JM, Dziewiatkowski J, Wojcik S, Sidor-Kaczmarek J, Morys J (2008) NPY-, SOM- and VIP-containing interneurons in postnatal development of the rat claustrum. Brain Res Bull 76:565-571.

Land PW, Simons DJ (1985) Cytochrome oxidase staining in the rat SmI barrel cortex. J Comp Neurol 238:225-235.

Li ZK, Takada M, Hattori T (1986) Topographic organization and collateralization of claustrocortical projections in the rat. Brain Res Bull 17:529-532.

Mathur BN, Caprioli RM, Deutch AY (2009) Proteomic analysis illuminates a novel structural definition of the claustrum and insula. Cereb Cortex 19:2372-2379.

Meng Z, Li Q, Martin JH (2004) The transition from development to motor control function in the corticospinal system. J Neurosci 24:605-614.

Minciacchi D, Molinari M, Bentivoglio M, Macchi G (1985) The organization of the ipsi- and contralateral claustrocortical system in rat with notes on the bilateral claustrocortical projections in cat. Neuroscience 16: 557-576.

Mitchinson B, Martin CJ, Grant RA, Prescott TJ (2007) Feedback control in active sensing: rat exploratory whisking is modulated by environmental contact. Proc Biol Sci 274:1035-1041.

Miyashita E, Keller A, Asanuma H (1994) Input-output organization of the rat vibrissal motor cortex. Exp Brain Res 99:223-232.

Mohapel P, Hannesson DK, Armitage LL, Gillespie GW, Corcoran ME (2000) Claustral lesions delay amygdaloid kindling in the rat. Epilepsia 41:1095-1101.

Mohapel P, Zhang X, Gillespie GW, Chlan-Fourney J, Hannesson DK, Corley SM, Li XM, Corcoran ME (2001) Kindling of claustrum and insular cortex: comparison to perihinal cortex in the rat. Eur J Neurosci 13:1501-1519.

Molinari M, Minciacchi D, Bentivoglio M, Macchi G (1985) Efferent fibers from the motor cortex terminate bilaterally in the thalamus of rats and cats. Exp Brain Res 57:305-312.

Olson CR, Graybiel AM (1980) Sensory maps in the claustrum of the cat. Nature 288:479-481.

Paxinos G, Watson C (2005) The rat brain in stereotaxic coordinates, Ed 6, New York: Academic.

Pearson RC, Brodal P, Gatter KC, Powell TP (1982) The organization of the connections between the cortex and the claustrum in the monkey. Brain res 234:435-441.

Reep RL, Corwin JV (2009) Posterior parietal cortex as part of a neural network for directed attention in rats. Neurobiol Learn Mem 91:104-113.

Reep RL, Corwin JV, Hashimoto A, Watson RT (1987) Efferent connections of the rostral portion of medial agranular cortex in rats. Brain Res Bull 19:203-221.

Reep RL, Wu JH, Cheatwood JL, Corwin JV, Kartje GL, Mir A (2008) Quantification of synaptic density in corticostriatal projections from rat medial agranular cortex. Brain Res 1233:27-34.

Reiner A, Jiao Y, Del Mar N, Laverghetta AV, Lei WL (2003) Differential morphology of pyramidal tract-type and intratelencephalically projectingtype corticostriatal neurons and their intrastriatal terminals in rats. J Comp Neurol 457:420-440.

Remedios R, Logothetis NK, Kayser C (2010) Unimodal responses prevail within the multisensory claustrum. J Neurosci 30:12902-12907.

Rouiller EM, Moret V, Liang F (1991) Comparison of the connectional properties of the two forelimb areas of the rat sensorimotor cortex: support for the presence of a premotor or supplementary motor cortical area. Somatosens Mot Res 10:269-289.

Sanderson KJ, Welker W, Shambes GM (1984) Reevaluation of motor cortex and of sensorimotor overlap in cerebral cortex of albino rats. Brain Res 292:251-260.

Sherk H (1986) The claustrum and the cerebral cortex. In: Cerebral cortex: sensory-motor areas and aspects of cortical connectivity (Jones EG, Peters A, eds), pp 467-499. New York: Plenum.

Sloniewski P, Usunoff KG, Pilgrim CH (1986) Retrograde transport of fluorescent tracers reveals extensive ipsi- and contralateral claustrocortical connections in the rat. J Comp Neurol 246:467-477.

Towal RB, Hartmann MJ (2006) Right-left asymmetries in the whisking behavior of rats anticipate head movements. J Neurosci 26:8838-8846.

Voigt T, De Lima AD, Beckmann M (1993) Synaptophysin immunohistochemistry reveals inside-out pattern of early synaptogenesis in ferret cerebral cortex. J Comp Neurol 330:48-64.

Wilson CJ (1986) Postsynaptic potentials evoked in spiny neostriatal projection neurons by stimulation of ipsilateral and contralateral neocortex. Brain Res 367:201-213.

Wilson CJ (1987) Morphology and synaptic connections of crossed corticostriatal neurons in the rat. J Comp Neurol 263:567-580. 